Various modifications of Theorem III may easily be secured. For example, in case we make the additional assumptions that the function $f(x, y)$ is bounded and is measurable in $y$ for each $x$, then the set $\mathbb{E}$ may be replaced by the interval $(a, b)$. These additional assumptions are fulfilled in particular if $f$ is bounded and Borel measurable on the square where it is defined. In this case the function $g(x, x)$ is Borel measurable on $(a, b)$. As another modifica-

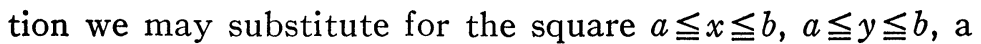
bounded measurable set $\xi_{0} \xi_{0}$, consisting of those points of the plane having $x$ and $y$ each in a linear measurable set $\mathbb{E}_{0}$. Then the integral is understood to be taken over those points of the interval $(a, x)$ contained in $\xi_{0}$.

HARVARD UNIVERSITY

\title{
A GENERAL THEORY OF REPRESENTATION OF FINITE OPERATIONS AND RELATIONS*
}

\author{
BY B. A. BERNSTEIN
}

Let $a \bmod n$ denote the least positive residue modulo $n$ of an integer $a$, i. e., the least positive integer obtained from $a$ by rejecting multiples of $n$. Consider the polynomials modulo a prime $p$

$$
\begin{gathered}
a_{0}+a_{1} x+\cdots+a_{p-1} x^{p-1}, \bmod p, \\
f_{0}(x)+f_{1}(x) y+\cdots+f_{p-1}(x) y^{p-1}, \bmod p,
\end{gathered}
$$

where in (1) $a_{i}$ are least positive $p$-residues and $x$ ranges over the complete system of least positive $p$-residues, and where (2) is a polynomial modulo $p$ in $y$ whose coefficients $f_{i}(x)$ are modular polynomials in $x$ of form (1). In a previous paper $\dagger$ I developed a theory of representation of abstract

* Presented to the Society, San Francisco Section, October 25, 1924.

$\dagger$ Proceedings of the International Mathematical Congress, TORONTO, 1924. 
binary operations and dyadic relations in a finite class of elements, in which theory the polynomials (2) entered fundamentally. I now wish to note the fact that this theory can be extended to finite operations and relations in general.

An $m$-ary operation $O$ in a class $K$ is a rule which determines for every ordered set of $m K$-elements $x_{1}, x_{2}, \cdots, x_{m}$ what $K$-element $O\left(x_{1}, x_{2}, \cdots, x_{m}\right)$, if any, corresponds to the set. If $K$ is finite, such a rule may always be given by an $m$-dimensional operation table or, when $m>1$, by $n$ tables each of $m-1$ dimensions. An $m$-adic relation $R$ in $K$ is a rule which states for every ordered set of $m$ $K$-elements $x_{1}, x_{2}, \cdots, x_{m}$ whether or not these elements should be associated together in a proposition $R\left(x_{1}, x_{2}, \cdots, x_{m}\right)$. When $K$ is finite, such a rule may always be given by an $m$-dimensional relation table or by $n$ tables each of $m-1$ dimensions $(m>1)$, in which the fact that $R\left(x_{1}, x_{2}, \cdots, x_{m}\right)$ holds may be indicated by "+" and that it does not hold by "-".* The function fundamental in the representation of finite $m$-ary operations and $m$-adic relations in general is the modular polynomial $f\left(x_{1}, x_{2}, \cdots, x_{m}\right)$ of the form

$$
f_{0}+f_{1} x_{m}+\cdots+f_{p-1} x_{m}^{p-1}, \bmod p,
$$

where $p$ is prime and the coefficients $f_{i}$ are polynomials modulo $p$ in the $m-1$ arguments $x_{1}, x_{2}, \cdots, x_{m-1}$. Noting that when the $K$-elements are $n$ in number they may be labeled $0,1, \cdots, n-1$, we may state the general theory by the propositions $A, B, C$ following.

Proposition A. Given an arbitrary set of least positive $n$-residues

$$
e_{0}, e_{1}, \cdots, e_{n-1}
$$

if and only if $n$ is prime, a function $f(x)$ of form (1) can always be obtained such that

$$
f(0)=e_{0}, \quad f(1)=e_{1}, \cdots, f(n-1)=e_{n-1} ;
$$

namely (1) in which (modulo $n$ )

* This " \pm " notation has been used by II. M. Sheffer. 
(6)

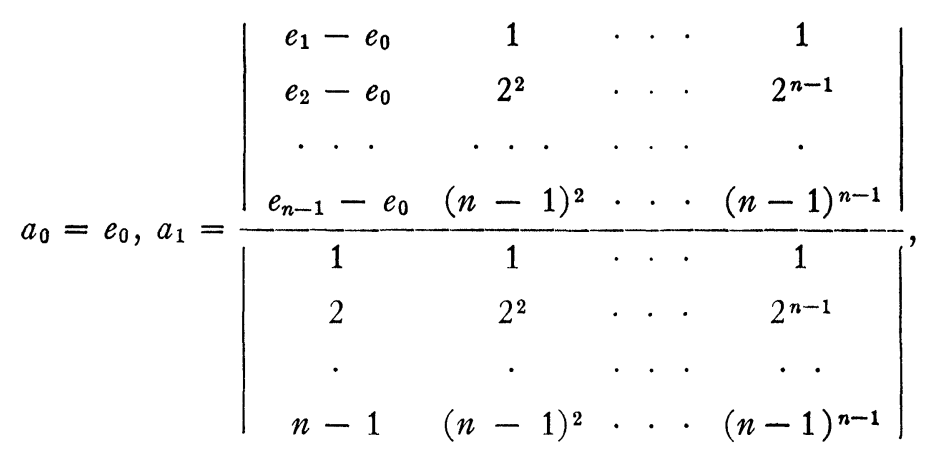

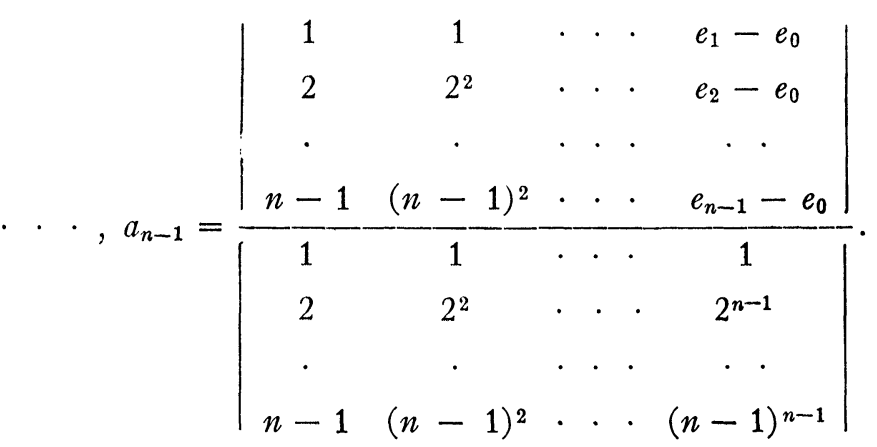

Proposition B. Given any $m$-dimensional operation table for the $K$-elements $0,1, \cdots, n-1$; a function equivalent to this table may be found having the form (7)

$$
f+0 / \varphi
$$

where $f$ and $\varphi$ are modular polynomials of form (3), with the $x$ 's ranging over the $K$-elements.

Proposition $C$. Given any $m$-dimensional relation table for the $K$-elements $0,1, \cdots, n-1$; an equation equivalent to this table may be found having the form

$$
f=0 \text {, }
$$

where $f$ is a modular polynomial of form (3), with the $x$ 's ranging over the $K$-elements.

For the proof of Proposition A the reader is referred to the paper cited above.

The second term of ( 7$)$ is designed to care for operations that do not satisfy the conditions of closure. To see the 
truth of Proposition B, consider several cases. (i) If $n$ is prime and the given operation satisfies the condition of closure, function ( 7 ) is the polynomial of form (3) obtained from the given table by repeated application of Proposition A. (ii) If $n$ is prime and the given operation does not satisfy the closure condition, consider the table got from the given table by assigning $K$-elements to the sequences $x_{1}, x_{2}, \cdots, x_{m}$, to which no $K$-element corresponds; consider also the table got from the given table by assigning 0 to each sequence to which no $K$-element corresponds and a $K$-element not 0 to any other sequence. The polynomials (3) equivalent to the derived tables will be respectively the $f$ and $\varphi$ of (7). (iii) If $n$ is composite, consider any operation table for a prime number $p(>n)$ of elements which will give the original table when the $x$ 's range over the $n$ elements of $K$; the function equivalent to this table, with the $x$ 's ranging over the $K$-elements, will be the required function (7).

To see that Proposition $\mathrm{C}$ is true, consider any operation table got from the given relation table by changing each "+" to 0 and each "-" to some $K$-element not 0 ; the function equivalent to this operation table will be the $f$ of (8).

Our theory of representation shows that any finite mathematical system, quantitative or non-quantitative, can be represented arithmetically (and geometrically). The theory also makes clear the nature of operations and relations, and it brings out the fact that an $m$-ary operation is the same as an $(m+1)$-adic relation, and $m$-adic relation the same as one or more $(m-1)$-ary operations $(m>1)$.*

UNIVERSITY OF CALIFORNIA

* For other applications and for various illustrations, see the above cited paper. See also this Bulletin, vol. 30 (1924), p. 24, and American JOURNAL, vol. 46 (1924), p. 110. 